Special Reviews

Juntendo Medical Journal

2014. 60 (4), 287-292

\title{
The Role of Smad4 in Murine T Cells in Terms of IgA Nephropathy and Potential Mechanism of Glomerular IgA Deposition
}

\author{
HIROYUKI INOSHITA*, YASUHIKO TOMINO* \\ *Division of Nephrology, Department of Internal Medicine, Juntendo University Faculty of Medicine, Tokyo, Japan
}

\begin{abstract}
A link between glomerular IgA nephropathy (IgAN) and T helper $2(\mathrm{Th} 2)$ response has been suggested; however, the mechanisms are poorly defined because of the lack of an appropriate model. Previously, we reported a novel murine model characterized by lineage-restricted deletion of the gene encoding MAD homologue 4

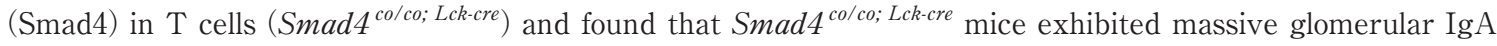
deposition, increased albumin creatinine ratio, aberrant glycosylated IgA, IgA complexed with IgG1 and IgG2a, and polymeric IgA, all known features of $\operatorname{IgAN}$ in humans. These findings indicate that Smad4 ${ }^{\text {co/co; Lck-cre }}$ mice could be a useful model for studying the mechanisms between IgAN and Th2 response, and furthermore, the disruption of Smad4-dependent signaling in T cells may play an important role in the pathogenesis of human IgAN and contribute to a Th2 T-cell phenotype.
\end{abstract}

Key words: IgA nephropathy, Smad4 signaling, regulatory T cell, Th2 cytokines

\section{Introduction}

$\operatorname{Ig} \mathrm{A}$ nephropathy (IgAN) is the most common form of glomerulonephritis throughout the world. The disease leads to progressive renal failure in a substantial proportion of patients. Despite an expanding international research effort over 40 years, the mechanisms of pathogenesis are still obscure. The hallmarks of this disease are hematuria, variable degrees of proteinuria, low grade proliferative glomerulonephritis and immune deposition of predominantly IgA in the renal mesangium. There is frequently dysregulation of circulating $\operatorname{Ig} \mathrm{A}$ in patients with $\operatorname{Ig} \mathrm{AN}$; notably, the elevated levels of IgA in serum are aberrantly glycosylated and biased towards higher molecular weight isoforms, and bound in complexes with IgG that are specific for truncated $O$-glycans ${ }^{1)-4)}$.

Skewed T helper 2 (Th2) cytokine production has been implicated in the pathogenesis of IgAN. Compared to other forms of glomerulonephritis, IgAN is more common in industrialized nations; this suggests that less exposure to microorganisms results in decreased T helper 1 (Th1) response and increased Th2 response ${ }^{5)}$. Moreover, patients with IgAN exhibit bias toward Th2 cytokine production during disease exacerbation, but heightened secretion of Th1 cytokines during remission ${ }^{6}$. Both human and murine B cells in vitro produce higher IgA levels in response to a mixture of recombinant interleukin (IL) -4 and IL-5 (Th2 cytokine) compared to control cultures, and mRNA levels of both core $1 \beta 1$,3-galactosyltransferase and its molecular chaperone Cosmc, as well as $\beta 1,3$-galactosyltransferase enzymatic activity are down-regulated by recombinant IL-4 in human B cells ${ }^{7)}$. These results suggest that skewed Th2 cytokine production leads to elevated levels of abnormally glycosylated IgA in the serum of patients with IgAN.

Recently, we generated conditional knockout mice in which the gene encoding Smad4 is deleted selectively in T cells ( $\operatorname{Smad} 4^{\text {co/co; Lck-cre }}$ ) by crossing mice homozygous for a Smad4 allele flanked with the lox sequence with mice expressing a transgene

Corresponding author: Hiroyuki Inoshita

Division of Nephrology, Department of Internal Medicine, Juntendo University Faculty of Medicine

2-1-1 Hongo, Bunkyo-ku,Tokyo 113-8421, Japan

TEL: +81-3-3813-3111 E-mail: ino-hi@juntendo.ac.jp

333rd Triannual Meeting of the Juntendo Medical Society “Medical Research Update” 〔Held on May 17, 2014〕

〔Received June 26, 2014] 
encoding a Cre recombinase driven by the $l c k$ promoter $^{8)}$. Interestingly, these $\operatorname{Smad} 4^{\text {co/co; Lck-cre }}$ mice have higher serum IgA levels than WT mice, and their $\mathrm{T}$ cells produce higher levels of Th2 cytokines (e. g. IL-4, IL-5, IL-6 and IL-13) when stimulated in vitro. In this article, we demonstrate our published data which shows that the Smad4 ${ }^{c o / c o ; ~ L c k-c r e ~ m i c e, ~ T h 2 ~ p r o n e ~ m i c e, ~ h a v e ~ I g A N-~}$ like phenotype ${ }^{9)}$ and propose a possible mechanism with new data in order to answer "how does IgA deposit to glomeruli?"

\section{Disruption of Smad4 expression in $T$ cells leads to IgA nephropathy-like manifestations}

\section{Predominant IgA deposition in glomeruli of} Smad4 $4^{\text {co/co; Lck-cre }}$ mice

By immunofluorescence, intense IgA deposits were detected in the glomerular mesangium in most $\mathrm{T}$ cell-specific Smad4 deficient (Smad4 $4^{\text {co/co; Lck-cre }}$ ) mice, whereas trivial IgA deposits were found in control, wild type $\left(\mathrm{Smad}_{4}^{+/+ \text {Lck-cre }}, \quad\right.$ Smad4 $4^{\text {co/co }}$; designated WT) mice (Figure-1A). Weak C3 deposits in mesangial areas and Bowman's capsule were detected in both Smad4 $4^{\text {co/co; Lck-cre }}$ and WT mice. By light microscopic examination of periodic acid-Schiff (PAS) stained sections, the kidneys of Smad4 ${ }^{\text {co/co; Lck-cre }}$ mice exhibited no marked abnormalities (Figure-1A). Specifically, mesangial proliferation, expansion of mesangial matrix, and other glomerular (or extraglomerular) changes were not detected in Smad4 ${ }^{\text {co/co; Lck-cre }}$ mice relative to WT mice.

\section{Glomerular function in Smad4 $4^{\text {co/co; Lck-cre }}$ mice}

Excretion of albumin in the urine, especially when expressed as an albumin to creatinine ratio (ACR), was significantly higher in Smad4 ${ }^{c o / c o ; ~ L c k-c r e ~ m i c e ~}$ than in controls (Figure-1B). Although there is no statistical difference between Smad4 $4^{\text {co/co; Lck-cre }}$ mice and controls, there was a trend toward an increase in urinary hemoglobin (after freeze-thaw, as a measure of hematuria) in Smad4 ${ }^{\text {co/co; Lck-cre }}$ mice when compared to controls (Figure-1B).

\section{Characteristics of circulating IgA in serum of Smad4 $4^{\text {co/co; Lck-cre }}$}

As reported previously ${ }^{8}$, the levels of $\operatorname{IgA}$ in the serum from Smad4 $4^{c o / c 0 ; \text { Lck-cre }}$ mice at 3 months of age were dramatically increased compared with agedmatched WT mice (Figure-2A). We next analyzed
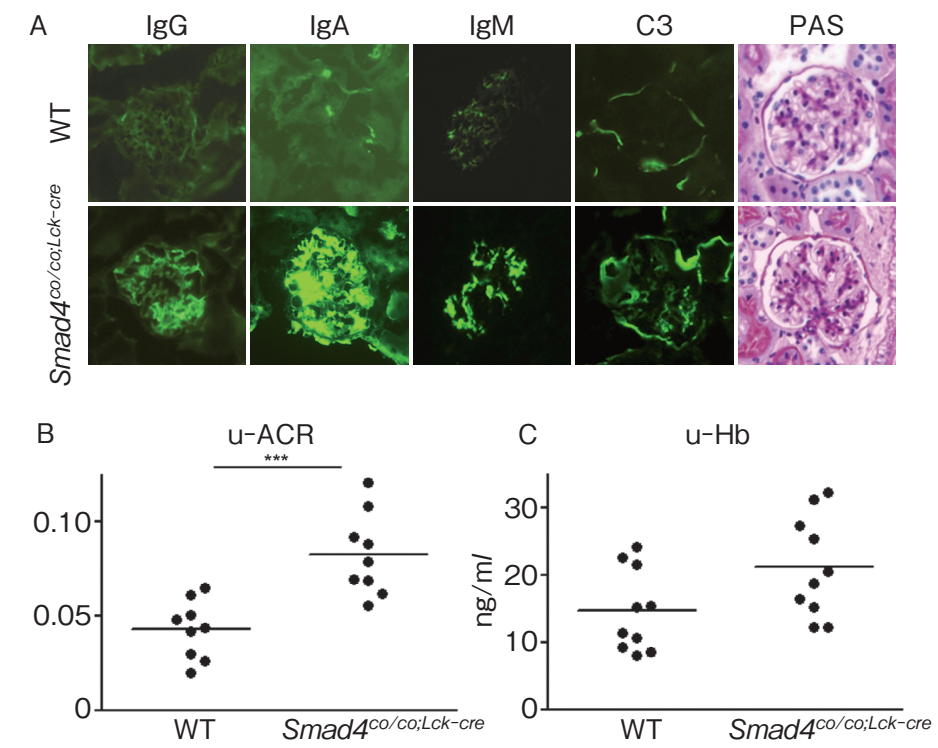

Figure-1 Deletion of Smad4 in T cell induced predominant IgA deposition in glomeruli and proteinuria A. Immunofluorecence studies showed glomerular deposition of predominantly IgA, with lesser amounts of $\operatorname{IgG}$ and IgM deposition in Smad $4^{c o / c o ;}$ Lck-cre mice compared with WT mice at 3 months of age. C3 deposition was occasionally observed in both mesangial areas and Bowman's capsule in Smad4 ${ }^{c o / c o}$; Lck-cre mice, but was restricted to Bowman's capsule in WT mice. PAS staining showed no significant difference between WT and Smad4 ${ }^{c o / c o}$; Lck-cre mice. Original magnification; x400. B. Increased albumin creatinine ratio (ACR) was observed in urines of Smad4 ${ }^{\text {co/co; Lck-cre }}$ mice $(\mathrm{n}=9)$ compared with WT mice $(\mathrm{n}=9)(0.043 \pm 0.005$ vs $0.083 \pm 0.007)$. C. There is no

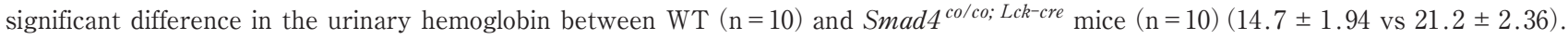
Data are expressed as the mean \pm SEM from one of three experiments with similar results; p values were calculated with Student's $t$ test. $* * * \mathrm{p}<0.001$ 
the pattern of glycosylation of $\operatorname{IgA}$; the relative levels of terminal sialic acid (Figure-2B) and terminal or penultimate galactose (Figure-2C) on serum $\operatorname{Ig} \mathrm{A}$ from Smad4 $4^{c o / c o ; ~ L c k-c r e}$ mice significantly decreased compared with those on the IgA from WT mice. By sandwich ELISA, the levels of complexes of IgG1-IgA and IgG2-IgA in serum from Smad $4^{\text {co/co; Lck-cre }}$ mice were significantly higher than those in WT mice (Figure-2, D and E). Finally, Western blotting revealed that the circulating $\operatorname{Ig} \mathrm{A}$ in Smad4 $4^{\text {co/co; Lck-cre }}$ mice is predominantly polymeric, whereas most of $\mathrm{IgA}$ from WT mice is monomeric (Figure-2F).

\section{4. mRNA levels of $\beta 4 \mathrm{GalTs}$ in B-cells from $\operatorname{Smad}^{\text {co/co; Lck-cre }}$ mice}

In mice, $\beta 1,4$-galactosyltransferases ( $\beta 4 \mathrm{GalT}$ ) are involved in the synthesis of IgA glycosylation, and seven $\beta 4 \mathrm{GalT}$ genes ( $\beta 4 \mathrm{GalT1}$ to 7 ) have been isolated so far. To determine which $\beta 4 \mathrm{GalT}$ is associated with the aberrant glycosylation, $\beta 4 \mathrm{GalT}$ (1 to 7) mRNA expressions in purified B cells from Smad4 ${ }^{c o / c o ; ~ L c k-c r e}$ mice and WT mice were analyzed by real-time RT-PCR using housekeeping GAPDH gene as the internal control. Real-time RT-PCR revealed that expression of mRNAs for $\beta 4 \mathrm{GalT} 2$ and 4 appeared to be significantly decreased in B-cells from Smad4 ${ }^{\text {co/co; Lck-cre }}$ mice as compared with B cells from WT mice (Figure-2G).
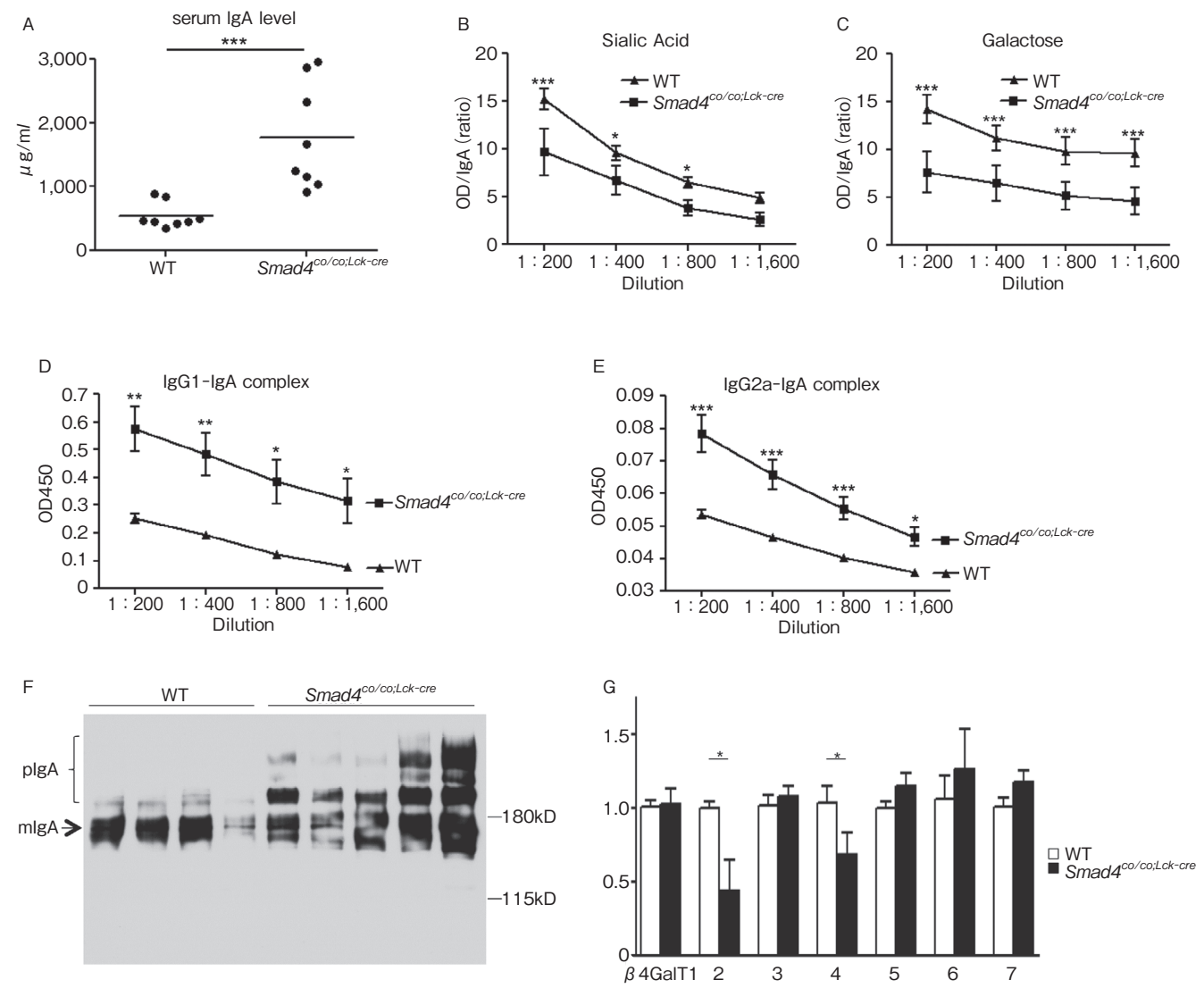

Figure-2 Characteristics of circulating IgA in mice lacking Smad4 in T-cells are similar to those in patients with IgA nephropathy

A. Markedly increased serum levels of IgA in Smad4 ${ }^{\text {co/co; } \text { Lck-cre }}$ mice $(\mathrm{n}=8)$ compared with WT mice ( $\left.\mathrm{n}=8\right)(1773 \pm 294.3 \mathrm{vs} 545.2 \pm$ 71.39). B and C. Aberrant glycosylation of circulating IgA from Smad4 ${ }^{c o / c o ; ~ L c k-c r e ~ m i c e ~}(\mathrm{n}=6$, black square) and WT mice $(\mathrm{n}=6$, black triangle) was examined by lectin ELISA. Both sialic acid and galactose on circulating IgA from the mutants were lower than those from

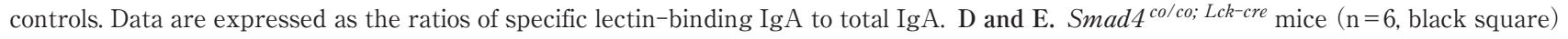
exhibit an increased in both IgG1-IgA complexes and IgG2a-IgA complexes in the circulation,compared with WT mice $(\mathrm{n}=6$, black triangle). Data are expressed as mean \pm SEM from one of three experiments with similar results; p values were calculated with $t$ test or Bonferroni post test. ${ }^{*} * \mathrm{p}<0.001,{ }^{*} \mathrm{p}<0.01$, and ${ }^{*} \mathrm{p}<0.05$. F. The predominance of polymeric IgA was observed in serum from Smad4 ${ }^{c o / c o}$; Lck-cre mice, but not in WT mice, as determined by Western blot. G. mRNA was isolated from purified B-cells (>95\% CD19+), and RT-PCR was performed using primers specific for $\beta 4 \mathrm{GalT1}$ to 7 , and GAPDH. $\beta 4 \mathrm{GalT} 2$ and $4 \mathrm{mRNA}$ expression in Smad4 ${ }^{\text {co/co; } L c k-c r e}$ mice ( $\mathrm{n}=4$, black column) were significantly reduced relative to controls $(n=4$, white column). All samples were normalized to GAPDH levels. Data are expressed as mean \pm SEM; p values were calculated with $t$ test. ${ }^{*} \mathrm{p}<0.05$ 


\section{$\beta$-actin as a potential target antigen specified by abnormal IgA in Smad4 ${ }^{\text {co/co; Lck-cre mice }}$}

The strong mesangial IgA deposition, albuminuria and presence of structural derangements in circulating IgA in Smad4 $4^{c o / c o ; ~ L c k-c r e ~}$ mice are evocative of human IgAN. One mechanism whereby the abnormal circulating IgA might accumulate in the mesangium and promote proteinuria posits that the IgA has autoimmune specificity for one or more antigens expressed by mesangial cells. Emergence of autoantibodies is more likely in hosts with defective function, such as the Smad $4^{c o / c o ; ~ L c k-c r e}$ mice. To assess this possibility in Smad4 $4^{\text {co/co; Lck-cre }}$ mice, we probed wild type mesangial cell lysates by Western blotting with sera from Smad4 $4^{\text {co/co; Lck-cre }}$ and WT mice. A robust protein band of approximately $43 \mathrm{kD}$, and a weaker $32 \mathrm{kD}$ band, were specifically detected by circulating $\operatorname{IgA}$ from Smad4 ${ }^{c o / c o ; ~ L c k-c r e ~ m i c e, ~ b u t ~ n o t ~ b y ~ c i r c u l a t i n g ~ I g A ~}$ from WT mice (Figure-3A). A band at approximately $82 \mathrm{kD}$ was identified by circulating IgA from
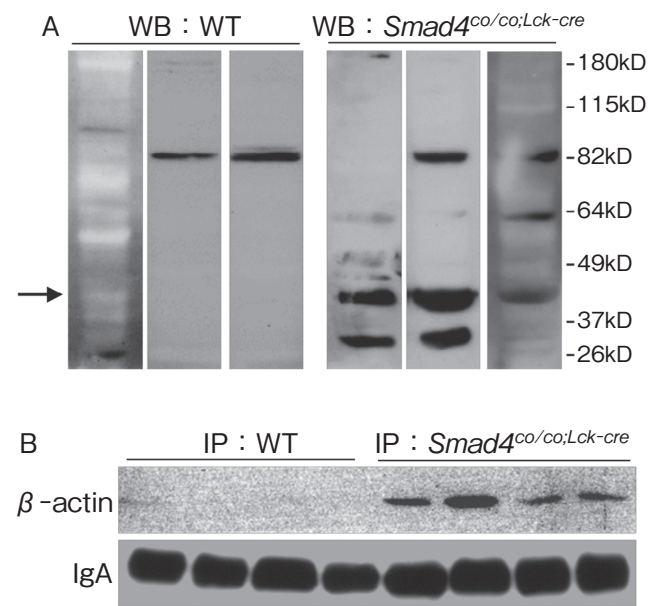

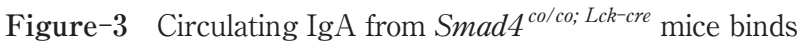
to $\beta$-actin in mesangial cells

A. Blots of mesangial cell lysate incubated with serum samples from Smad4 ${ }^{c o / c o ; ~ L c k-c r e ~(r i g h t) ~ o r ~ W T ~ m i c e ~(l e f t), ~ f o l l o w e d ~ b y ~}$ anti-mouse IgA antibody. A consistent band at approximately 43 $\mathrm{kD}$ (arrow) in mouse mesangial cell lysate was identified by circulating IgA from the mutants, but not controls. B. Mixtures of mesangial cell lysate and serum samples from either Smad4 $4^{c o / c o ; ~ L c k-c r e}$ or WT mice were immunoprecipitated with anti-mouse IgA-Sepharose. The immunoprecipitates were then separated by on SDS-PAGE, under reducing conditions and blotted onto nitrocellulose. The membrane was incubated with anti- $\beta$-actin (top) or anti-mouse $\operatorname{IgA}$ (bottom) antibody. The ( $43 \mathrm{kD}$ ) bands detected by anti- $\beta$-actin antibody were present only in Lanes loaded with immunoprecipitates formed with serum from Smad4 $4^{\text {co/co; Lck-cre }}$ mice. both Smad4 $4^{\text {co/co; Lck-cre }}$ and WT mice. The $43 \mathrm{kD}$ band was excised from the gels and subjected to in-gel digestion followed by mass spectroscopy; the most probable mesangial cell protein target is $\beta$-actin. Accordingly, wild type mesangial cell lysate was subject to immunoprecipitation with serum from either Smad4 $4^{c o / c o ; \text { Lck-cre }}$ or WT mice, using antimouse IgA conjugated to Sepharose. Subsequently, the precipitates were separated by electrophoresis and probed by Western blotting using antibodies specific for $\beta$-actin and IgA. As shown in Figure$3 \mathrm{~B}$, the anti- $\beta$-actin antibody stained a band of 43 $\mathrm{kD}$ in lanes loaded with immunoprecipitates from Smad4 $4^{\text {co/co; Lck-cre }}$ mice, but this band was not found in immunoprecipitates from WT mice. Furthermore, by double immunofluorescence, expression of $\beta^{-}$ actin was confined to glomeruli of both Smad4 $4^{\text {co/co; Lck-cre }}$ and WT mice, whereas co-localization of $\operatorname{Ig} \mathrm{A}$ with $\beta$-actin was only observed in glomeruli of Smad4 $4^{c o / c o ; ~ L c k-c r e}$ mice, and not in those

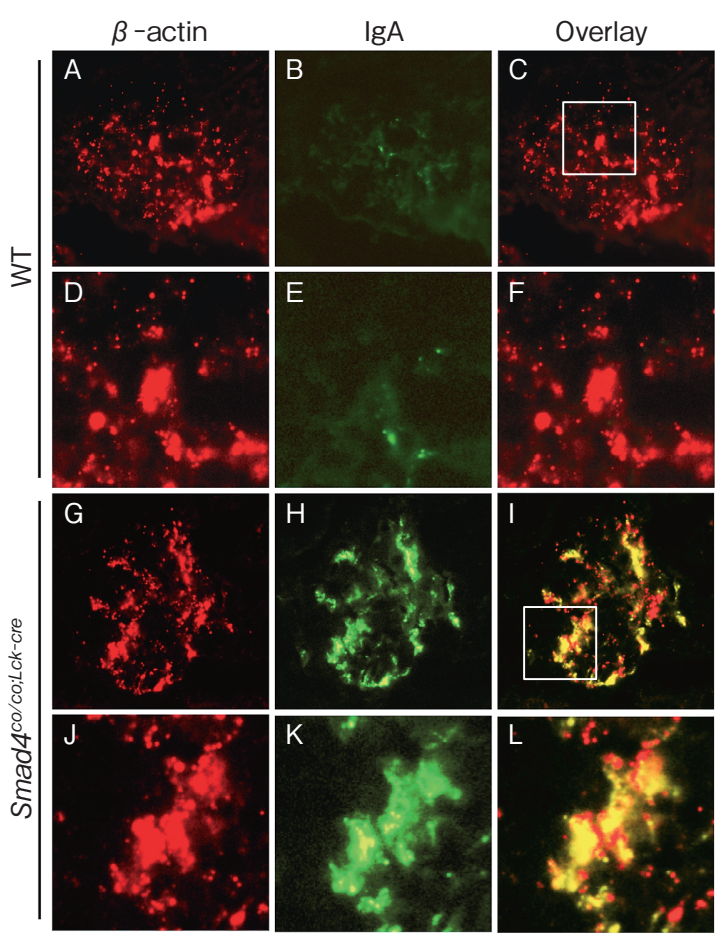

Figure-4 Glomerular IgA deposition was colocalized with $\beta$-actin in glomeruli from Smad4 ${ }^{\text {co/co; Lck-cre mice }}$ Double immunofluorescent staining with (A, D, G, and J, red) $\beta$-actin and (B, E, H, and $\mathrm{K}$, green) IgA was performed to evaluate the colocalization in glomeruli from (G-L) Smad4 $4^{\text {co/co; Lck-cre }}$ and (A-F) WT mice. Magnified images are $\mathrm{D}-\mathrm{F}$ and $\mathrm{J}-\mathrm{L}$. The staining of $\beta$-actin is limited in glomeruli from both ( $\mathrm{G}$ and $\mathbf{J}$ ) the mutant and ( $\mathrm{A}$ and $\mathrm{D}$ ) controls. Overlaid images show colocalization of IgA deposition with $\beta$-Actin in glomeruli from (I and L) the Smad4 $4^{\text {co/co; Lck-cre }}$, but not $(\mathrm{C}$ and F) WT mice. Original magnification; $\mathrm{x} 400$ 
of WT mice (Figure-4).

\section{Discussion}

In our studies, Smad4 $4^{\text {co/co; Lck-cre }}$ mice develop mesangial IgA deposits associated with increased urinary albumin excretion and dysregulation of $\operatorname{Ig} \mathrm{A}$ synthesis. The latter is manifest by increased serum IgA with aberrant glycosylation that includes an elevated proportion of polymer and that binds to $\operatorname{IgG}$ to form mixed isotype complexes. These are features in common with IgA isolated from patients with IgAN. In addition, we investigated a potential mechanism of glomerular $\operatorname{Ig} \mathrm{A}$ deposition in these mutant mice.

Smad4 is a key molecule that influences $\mathrm{T}$ cell differentiation; as part of a complex with Smad2 and Smad3, Smad4 serves as a transcription factor for forkhead box P3 (Foxp3), driving the expression of genes important for the genesis of regulatory T cells (Tregs). Although the Smad4 $4^{\text {co/co; Lck-cre mice }}$ represent a useful model for the investigation of the balance between Th2 and Tregs, the means by which deletion of Smad4 promotes bias towards Th2 function remains unclear. A reciprocal relationship between Th2 cells and Tregs is recognized. The transcriptional activator STAT6 enhances the expression of the master regulator of Th2 differentiation, GATA3. STAT6 and GATA3 synergize with STAT5 activation to induce the secretion of Th2 cytokines by activated Th2 cells. Apparently, Foxp3 can bind directly to GATA3; engagement in this heterodimeric complex diminishes the activity of each binding partner. The loss of Smad4 would likely diminish Foxp3, freeing GATA3 from inhibition while simultaneously impairing differentiation of Tregs. Indeed, a progressive decline in mucosal Treg has been described in the Smad4 $4^{\text {co/co; Lck-cre }}$ mice and in Smad3-/- mice ${ }^{10)}$. Upon stimulation of $\mathrm{T}$ cells, overproduction of Th2 cytokines, unopposed by Tregs, might lead to exaggerated production of $\operatorname{Ig} \mathrm{A}$ that bears aberrant glycans. Several reports indicate that, in vitro and in vivo, Th2 cytokines lead to increased production and defective glycosylation of $\operatorname{IgA}$. This interpretation is also supported by a recent report showing that upon OVA challenge, glomerular IgA deposition was detected in only double-transgenic mice that overexpress GATA3 and the ovalbumin (OVA) -specific T cell receptor ${ }^{11)}$.

The phenotype of Smad4 $4^{c o / c o ; \text { Lck-cre }}$ mice is reminiscent of human IgAN and suggests that loss of Smad4-signaling in $\mathrm{T}$ cells associates with $\operatorname{IgA}$ deposition in the mesangial area, dysregulation of $\operatorname{Ig} \mathrm{A}$, and proteinuria. However, there are some discrepancies between Smad4 ${ }^{\text {co/co; Lck-cre }}$ mice and spontaneous IgAN in humans. Notably, strong C3 deposition associated with mesangial IgA, morphological changes in glomeruli and hematuria are generally observed in human IgAN, but were not observed in Smad4 $4^{c o / c o ; ~ L c k-c r e}$ mice. These discrepancies may represent requirements beyond glomerular IgA deposition, such as complement activation and/or inflammatory mediators, for evolution of hematuria and/or morphologic expression of glomerular injury. Recently, a genome-wide association study (GWAS) of human IgAN identified a major IgAN susceptibility locus within the complement factor $\mathrm{H}$ gene $(\mathrm{CFH})$ cluster at chromosome 1q32. As $\mathrm{CFH}$ regulates complement activation through inhibition of the C3 convertase, the GWAS suggests that dysregulation of complement activation could be one of the mechanisms underlying progressive glomerular dysfunction. In addition to relatively minor glomerular complement deposition in spite of severe $\operatorname{IgA}$ deposition, the lack of hematuria and morphologically evident glomerular injury in Smad4 ${ }^{c o / c o \text {; Lck-cre }}$ mice may be explained by the strain of these knockout mice. According to reports in different murine models, glomerular dysfunction caused by $\operatorname{IgA}$ deposition is more pronounced in the $\mathrm{BALB} / \mathrm{c}$ strain compared to other backgrounds despite similar mesangial deposition of $\operatorname{Ig} \mathrm{A}$ and $\mathrm{C} 3{ }^{12)}$. Although the basis for these strain differences is unclear, we expect that deletion of Smad4 in BALB/c mice would lead to more morphologic and functional evidence of glomerular injury than observed in the current $(\mathrm{C} 57 \mathrm{BL} / 6 \times$ SvEv129 × FVB) strain.

Understandings of mechanisms of immune deposit formation are very important to elucidate processes of glomerular injury in glomerular diseases because most of glomerular diseases manifest various immune depositions such as IgG, IgA, IgM, C1q, C3, C4, C5, and mannose binding lectin in the glomeruli. Mechanism (s) of glomerular immune deposits formation are generally divided by three categories that (1) Circulating 
immune complex trapping; (2) In situ immune deposit formation exogenous antigens; (3) In situ immune deposit formation endogenous antigens. To examine the immune deposit formation, we focused on the third mechanism. Recent progress in proteomics technology allows us to discover new target antigens readily. For example, the M-type phospholipase $A_{2}$ receptor $\left(\mathrm{PLA}_{2} \mathrm{R}\right)$ in podocytes was identified by proteomics as a target antigen in $70 \%-80 \%$ of patients with idiopathic membranous nephropathy ${ }^{13)}, 20$ years after "megalin” was identified as the target antigen in podocytes in a membranous nephropathy rat model (Heymann's nephritis). Although no single pathogenic antigen is recognized by circulating $\operatorname{Ig} \mathrm{A}$ in human $\operatorname{Ig} \mathrm{AN}$, actin has been implicated in patients with IgAN. In the present study, we showed reactivity of serum $\operatorname{IgA}$ from Smad4 ${ }^{\text {co/co; Lck-cre }}$ mice with a dominant $43 \mathrm{kD}$ protein from an extract of mesangial cells; proteomic analysis indicated that $\beta$-actin is the likely target antigen. Immunoprecipitation and double immunofluorescence experiments support the conclusion that circulating IgA against $\beta$-actin exists in serum from Smad4 ${ }^{\text {co/co; Lck-cre }}$ mice. $\beta$-actin is a moderate-sized protein consisting of $\approx 375$ amino acids and is very highly conserved in most of cells. Cytoskeletal actins, such as $\beta$-actin, are involved in the structural integrity and motility of cells; actin interacts and associates with a number of other proteins, including actin-binding proteins/calcium associated proteins, are essential for controlling and regulating actin filament assembly. The binding of specific $\operatorname{Ig} \mathrm{A}$ to $\beta$-actin might alter cytoskeletal signal transduction and thereby interfere with cell functions. Immunofluorescence findings for Smad4 $4^{c o / c o ; ~ L c k-c r e}$ mice show dominant mesangial IgA deposition. $\beta$-actin is ubiquitous, the specific IgA to $\beta$-actin in Smad4 $4^{\text {co/co; Lck-cre }}$ mice may deposit to mesangial cells as well as podocytes and endothelial cells. It is tough question that why the specific IgA is tended to deposit to mesangial cells, balance between deposition and clearance of $\operatorname{Ig} \mathrm{A}$ in each cell may be attributed.

We conclude that Smad4 $4^{\text {co/co; Lck-cre }}$ mice produce heightened amounts of $\operatorname{IgA}$ that is mostly polymeric, aberrantly glycosylated, and complexed with IgG. These mice develop mesangial IgA deposits and proteinuria, reminiscent of human IgAN. In addition, we recognized that some of the circulating
$\operatorname{IgA}$ in Smad4 ${ }^{c o / c o ; ~ L c k-c r e ~ m i c e ~ h a s ~ s p e c i f i c i t y ~ f o r ~} \beta^{-}$ actin expressed in mesangial cells. Smad4 $4^{\text {co/co; Lck-cre }}$ mice could therefore provide us with new insights into the role that skewed Th2 cytokine production plays in altering circulating $\operatorname{Ig} \mathrm{A}$ and/or formation of mesangial immune deposits. Ultimately, these insights might promote the development of a new therapeutic strategy for IgAN.

\section{References}

1) van Es LA, van den Wall Bake AW, Valentijn RM, Daha MR: Composition of IgA-containing circulating immune complexes in IgA nephropathy. Am J Kidney Dis, 1988; 12: $397-401$.

2) Couser WG: Glomerulonephritis. Lancet, 1999; 353: 15091515.

3) Hiki Y, Odani H, Takahashi M, et al: Mass spectrometry proves under-O-glycosylation of glomerular IgA1 in IgA nephropathy. Kidney Int, 2001; 59: 1077-1085.

4) Barratt J, Smith AC, Molyneux K, Feehally J: Immunopathogenesis of IgAN. Semin Immunopathol, 2007; 29: 427-443.

5) Johnson RJ, Hurtado A, Merszei J, Rodriguez-Iturbe B, Feng L: Hypothesis: dysregulation of immunologic balance resulting from hygiene and socioeconomic factors may influence the epidemiology and cause of glomerulonephritis worldwide. Am J Kidney Dis, 2003; 42: $575-581$.

6) Scivittaro V, Ranieri E, Di Cillo M, Aventaggiato L, Emancipator SN, Schena FP: In vitro immunoglobulin production in relatives of patients with IgA nephropathy. Clin Nephrol, 1994; 42: 1-8.

7) Yamada K, Kobayashi N, Ikeda T, et al: Down-regulation of core 1 betal,3-galactosyltransferase and Cosmc by Th2 cytokine alters O-glycosylation of IgA1. Nephrol Dial Transplant, 2010; 25: 3890-3897.

8) Kim BG, Li C, Qiao W, et al: Smad4 signalling in T cells is required for suppression of gastrointestinal cancer. Nature, 2006; 441: 1015-1019.

9) Inoshita H, Kim BG, Yamashita M, et al: Disruption of Smad4 expression in T cells leads to IgA nephropathylike manifestations. Plos One, 2013; 8: e78736.

10) Nolting J, Daniel C, Reuter S, et al: Retinoic acid can enhance conversion of naive into regulatory $\mathrm{T}$ cells independently of secreted cytokines. J Exp Med, 2009; 206: 2131-2139.

11) Suzuki Y, Tomino Y: Potential immunopathogenic role of the mucosa-bone marrow axis in IgA nephropathy: insights from animal models. Semin Nephrol, 2008; 28: 66-77.

12) Chintalacharuvu SR, Yamashita M, Bagheri N, et al: T cell cytokine polarity as a determinant of immunoglobulin A ( $\operatorname{Ig} \mathrm{A})$ glycosylation and the severity of experimental IgA nephropathy. Clin Exp Immunol, 2008; 153: 456-462.

13) Beck LH Jr, Bonegio RG, Lambeau G, et al: M-type phospholipase A2 receptor as target antigen in idiopathic membranous nephropathy. N Engl J Med, 2009; 361: 11-21. 